\title{
MicroRNA-422a functions as a tumor suppressor in glioma by regulating the Wnt/ $\beta$-catenin signaling pathway via RPN2
}

\author{
JIKUI SUN ${ }^{1,2^{*}}$, ZHIJUAN CHEN $^{3,4 *}$, JINBIAO XIONG $^{3,4^{*}}$, QIONG WANG $^{2}$, FAN TANG $^{5}$, \\ XUEBIN ZHANG ${ }^{5}$, LIDONG MO ${ }^{2}$, CHEN WANG $^{2}$, WEIJIA FAN ${ }^{2}$ and JINHUAN WANG ${ }^{1,2}$ \\ ${ }^{1}$ School of Medicine, Nankai University, Tianjin 300071; ${ }^{2}$ Tianjin Cerebral Vascular and Neural \\ Degenerative Disease Key Laboratory, Tianjin Neurosurgery Institute, Department of Neurosurgery, \\ Tianjin Huan Hu Hospital, Tianjin 300350; ${ }^{3}$ Clinical Medicine School, Tianjin Medical University, Tianjin 300070; \\ ${ }^{4}$ Department of Neurosurgery, Tianjin Medical University General Hospital, Tianjin Neurological Institute, \\ Tianjin 300052; ${ }^{5}$ Pathology Department, Tianjin Huan Hu Hospital, Tianjin 300350, P.R. China
}

Received February 18, 2020; Accepted July 16, 2020

DOI: 10.3892/or.2020.7741

\begin{abstract}
MicroRNAs (miRs), which act as crucial regulators of oncogenes and tumor suppressors, have been confirmed to play a significant role in the initiation and progression of various malignancies, including glioma. The present study analyzed the expression and roles of miR-422a in glioma, and reverse transcription-quantitative PCR confirmed that miR-422a expression was significantly lower in glioblastoma multiforme (GBM) samples and cell lines compared with the low-grade glioma samples and the $\mathrm{H} 4$ cell line, respectively. miR-422a overexpression suppressed proliferation and invasion, and induced apoptosis in LN229 and U87 cell lines. Luciferase reporter assay, western blotting and RNA immunoprecipitation analysis revealed that ribophorin II (RPN2) is a direct functional target of miR-422a. Additionally, the overexpression of RPN2 partially reversed the miR-422a-mediated inhibitory effect on the malignant phenotype. Mechanistic investigation demonstrated that the upregulation of miR-422a inhibited $\beta$-catenin/transcription factor 4 transcriptional activity, at least partially through RPN2, as indicated by in vitro and in vivo experiments. Furthermore, RPN2 expression was inversely correlated with miR-422a expression in GBM specimens and predicted patient survival in the Chinese Glioma Genome Atlas, UALCAN, Gene Expression Profiling Interactive Analysis databases. In conclusion, the present data reveal a new
\end{abstract}

Correspondence to: Professor Jinhuan Wang, Tianjin Cerebral Vascular and Neural Degenerative Disease Key Laboratory, Tianjin Neurosurgery Institute, Department of Neurosurgery, Tianjin Huan Hu Hospital, Jizhao Road, Tianjin 300350, P.R. China

E-mail:wangjinhuan16@163.com

*Contributed equally

Key words: microRNA-422a, ribophorin II, Wnt//-catenin, glioma, apoptosis
miR-422a/RPN2/Wnt/ $\beta$-catenin signaling axis that plays critical roles in glioma tumorigenesis, and it represents a potential therapeutic target for GBM.

\section{Introduction}

Glioblastoma multiforme (GBM), an incurable primary brain tumor with a poor prognosis, is characterized by various genetic alterations, such as mutation of isocitrate dehydrogenase (IDH)1/2, amplification of epidermal growth factor receptor (EGFR) and dysregulation of multiple signaling pathways, such as the PI3K/AKT/mTOR, Wnt/ $\beta$-catenin and $\mathrm{NF} / \kappa \mathrm{B}$ pathways $(1,2)$. The high degree of heterogeneity based on this complicated molecular network in GBM is responsible for its poor treatment response, which is influenced by chemoand radiotherapy resistance (3). Despite current progress in high-throughput data containing more significant subtype genes identifications based on The Cancer Genome Atlas (TCGA) $(1,4,5)$, the precise molecular mechanism remains poorly understood. Previously, non-coding RNAs, including microRNAs (miRNAs or miRs) have been implicated in GBM and have been shown to form extensive complex crosstalk networks to participate in GBM initiation and development $(6,7)$; therefore, further identification of relevant complex signaling networks is urgently required to provide insights into combinational targeted therapy and treatment resistance.

miRNAs regulate gene expression by primarily binding to the 3'-untranslated region of target mRNAs, leading to translational inhibition or mRNA destabilization, which is extensively implicated in numerous cancers (8). Evidence suggests that miR-422a downregulation is closely associated with poor prognosis and unfavorable clinicopathologic parameters. For example, the miR-422a expression level is negatively associated with pathological grade, recurrence and metastasis in hepatic cell carcinoma (9), and has also been reported to predict lymphatic metastasis with high diagnostic accuracy in lung cancer (10). Additionally, serum miR-422a is regarded as a biomarker for the early diagnosis of colorectal adenocarcinoma (11). Furthermore, miR-422a, in combination with 
multiple key target genes, such as forkhead box Q1 (FOXQ1) and S-phase kinase associated protein 2 , has been extensively identified to act as a crucial tumor suppressor and prognostic factor during cancer progression and development in solid malignancies, including in nasopharyngeal carcinoma (12) and in retinoblastoma (13). The decreased expression of miR-422a and the identification of certain targets, such as PIK3CA, have been reported in glioma (14); however, the molecular mechanism of the miR-422a-mediated inhibitory effect on the glioma malignant phenotype is poorly understood and requires further investigation.

Ribophorin II (RPN2), which belongs to the key part of the oligosaccharyltransferase complex, is responsible for the $\mathrm{N}$-glycosylation of multiple proteins, and its glycosylation alterations have been confirmed to be associated with GBM malignant progression (15-17). Accumulating evidence has demonstrated that aberrant RPN2 overexpression is frequently associated with multiple clinical parameters, such as lymphatic metastasis, pathological grade and poor prognosis in a variety of tumors, including osteosarcoma, non-small cell lung cancer, advanced gastric cancer and colorectal cancer $(16,18-20)$. However, to the best of our knowledge, there is no report that indicates a relationship between miR-422a and RPN2 in glioma.

The present study initially investigated the miR-422a downregulation in GBM tissues and cell lines, and verified its negative relationship with the World Health Organization (WHO) grade. Additionally, overexpression of miR-422a markedly suppressed cell proliferation and invasion, and promoted apoptosis and cell cycle arrest at the G0/G1 phase. Mechanistically, miR-422a inhibited the Wnt/ $\beta$-catenin signaling pathway, as indicated by TOP/FOP luciferase and western blot assays. Furthermore, it was demonstrated that RPN2 was a direct functional target of miR-422a and plays significant roles in miR-422a-mediated inhibitory effects on Wnt/ $\beta$-catenin signaling, as well as the malignant phenotype. Therefore, the present study explored a novel potential axis involving miR-422a/RPN2/ $\beta$-catenin, which represents a novel therapeutic target for GBM.

\section{Materials and methods}

Patients and samples. A total of 39 glioma samples undergoing craniotomy for tumor were obtained from 15 male and 24 female patients (age range, 9-76 years; mean age, $42.4 \pm 16.2$ years) who were diagnosed by surgeons and pathologists at Tianjin Huan Hu Hospital (Tianjin, China) between January 2010 and June 2018. Among the 10 low-grade gliomas, 6 were WHO grade I (pilocytic astrocytoma) and 4 were WHO grade II (diffuse astrocytoma). All 29 GBM samples were WHO grade IV. None of the patients had received any radiotherapy, chemotherapy or any other anticancer treatments prior to surgery. Five normal adult brain tissue samples were collected from 4 males and 1 female patient (age range, 58-74 years; mean age, $66 \pm 6.3$ years) undergoing post-trauma surgery for severe traumatic brain injury. All the collected tissues were frozen immediately in liquid nitrogen and stored at $-80^{\circ} \mathrm{C}$. This study was approved by the Institutional Review Board of Tianjin Huan Hu Hospital, and written informed consent was obtained from all participants or the parents of patients below the legal age. Gene expression data from the mRNAseq_325 dataset and the relevant clinicopathological variables dataset were obtained from the Chinese Glioma Genome Atlas (CGGA) database (http://www.cgga.org.cn/), which is a web application for data storage and analysis to explore brain tumor datasets of $>2,000$ samples from Chinese cohorts (21). In addition, the 'Survival Map' module from the online databases Gene Expression Profiling Interactive Analysis 2 (GEPIA2; http://gepia.cancer-pku.cn/index.html), which is an interactive website that includes information for 514 low-grade and 162 high-grade glioma tissues obtained from TCGA and GTEx projects (22), was used to evaluate the relationships between RPN2 expression and overall survival and disease free survival. Moreover, UALCAN (http://ualcan. path.uab.edu) (23), which is a comprehensive interactive database containing data of 513 low-grade gliomas (248 grade II and 265 grade III) from The Cancer Genome Atlas, was also utilized to analyze RPN2 expression level in gliomas with different grades, and the association with the patient prognosis.

Cell culture and transfection. The human glioblastoma cell lines U87, U251 and A172 and the low-grade glioma cell line H4 were obtained from the Peking Union Medical College Cell Library. Human LN308, LN229 and T98G glioblastoma cells were obtained from the China Academia Sinica Cell Repository. The SNB19 cell line was purchased from iCell Bioscience, Inc. U87 and SNB 19 cell lines were authenticated using short tandem repeat profiling analysis. The U87 cell line (glioblastoma of unknown origin) used in the present study was of the American Type Culture Collection type. The cells were maintained in Dulbecco's modified Eagle's medium (Gibco; Thermo Fisher Scientific, Inc.) supplemented with $10 \%$ fetal bovine serum (Gibco; Thermo Fisher Scientific, Inc.) and 1\% penicillin/streptomycin (Invitrogen; Thermo Fisher Scientific, Inc.). All cell lines were incubated at $37^{\circ} \mathrm{C}$ with $5 \% \mathrm{CO}_{2}$.

The oligonucleotide sequences of the human miR-422a mimic and miRNA-negative control (miR-NC), and RPN2 overexpression plasmid and empty vector were purchased from Shanghai GenePharma Co., Ltd. The human miR-422a sequence was 5'-ACUGGACUUAGGGUCAGAAGGC-3', and the scrambled sequence was 5'-UUGUACUACACAAAAGUA CUG-3'. Prior to transfection, U87 and LN229 cells were incubated in a 6 -well plate at a density of $2 \times 10^{5}$ cells/well and then co-transfected with 200 pmol miR-422a mimic or miR-NC and RPN2 overexpression plasmid or empty vector $(2 \mu \mathrm{g})$ using X-tremeGENE transfection reagent (Roche Diagnostics) according to the manufacturer's protocol. Subsequent experiments were performed after $72 \mathrm{~h}$.

For RPN2-knockdown, the shRNA lentiviral vector targeting RPN2 (shRPN2; 5'-GGATCGCCCTTTCACAAA $\mathrm{T}-3^{\prime}$ ) and lentiviral vector negative control (sh-NC; 5'-TTC TCCGAACGTGTCACGT-3') were also purchased from Shanghai GenePharma Co., Ltd. LN229 cells were infected at a multiplicity of infection of 10 in the presence of $5 \mu \mathrm{g} / \mathrm{ml}$ polybrene (Shanghai GenePharma Co., Ltd.), following the manufacturer's instructions. Western blotting was performed to identify the knockdown efficiency after $72 \mathrm{~h}$.

Reverse transcription-quantitative PCR (RT-qPCR). Total RNA was isolated from glioma tissues and cells using TRIzol 
reagent (Thermo Fisher Scientific, Inc.), reverse transcribed to cDNA using PrimeScript RT Master mix (Takara Bio, Inc.), according to the manufacturer's instructions and subsequently qPCR was performed using TB Green Premix EXTaq II kit (Takara Bio, Inc.) for analysis of the mRNA expression of RPN2, TCF4, c-myc and cyclinD1. The amplification conditions were $95^{\circ} \mathrm{C}$ for $5 \mathrm{sec}$, followed by 40 cycles at $60^{\circ} \mathrm{C}$ for $30 \mathrm{sec}$ and $50^{\circ} \mathrm{C}$ for $30 \mathrm{sec}$. To analyze miRNA-422a expression, stem-loop RT was performed with an miScript PCR starter kit (Qiagen $\mathrm{GmbH}$ ) according to the manufacturer's instructions. qPCR was performed using miScript SYBR Premix Green PCR kit (Qiagen GmbH) and Roche LC480 quantitative Real-Time PCR system (Roche Diagnostics). The amplification conditions were $95^{\circ} \mathrm{C}$ for $15 \mathrm{~min}$, followed by 40 cycles at $94^{\circ} \mathrm{C}$ for $15 \mathrm{sec}$ and $55^{\circ} \mathrm{C}$ for $30 \mathrm{sec}$. GAPDH or U6 levels were selected as internal controls for mRNA and miRNA expression, respectively, and fold changes were calculated using the $2^{-\Delta \Delta \mathrm{Cq}}$ method (24). Data were analyzed from three independent experiments and are presented as the mean \pm standard deviation. The primer sequences for the detection of mRNAs and miR-422a expression were as follows: RPN2 forward, 5'-CTCTGACGCCCACTCACT AC-3' and reverse, 5'-AATAGAGATCTTTGCATCTGG CAC-3'; TCF4 forward, 5'-CAAATAGAGGAAGCGGGG C-3' and reverse, 5'-TGCTGAGAGAGATGGAGGAGA-3'; cyclinD1 forward, 5'-AACTACCTGGACCGCTTCCT-3' and reverse, 5'-CCACTTGAGCTTGTTCACCA-3'; c-myc forward, 5'-TTCGGGTAGTGGAAAACCAG-3' and reverse, 5'-CAGCAGCTCGAATTTCTTCC-3'; GAPDH forward, 5'-CATGAGAAGATGACAACAGCCT-3' and reverse, 5'-AGTCCTTCCACGATACCAAAGT-3'; miR-422a forward, 5'-GGGTCAGAAGGCCTGAGTCT-3' and reverse, 5'-CAA AGCTTGGCTCAGGGACA-3'; and U6 forward, 5'-CTC GCTTCGGCAGCACA-3' and reverse, 5'-AACGCTTCA CGAATTTGCGT-3'.

Dual-luciferase reporter assay. The PicTar (http://www. pictar.org/), miRmap (http://miRNAMap.mbc.nctu.edu.tw/.) and miRanda (http://www.microrna.org/microrna/home.do) databases were applied to identify targets of miR-422a, and the seed sequence of miR-422a was identified to matched the 3'-untranslated region (3'UTR) of the RPN2 gene. The 3'UTR of RPN2 containing the miR-422a binding site and corresponding mutant site were inserted into the pMIR-REPORT vector (Promega Corporation). LN229 and U87 cells (1x10\%/well) were cultured in 96-well plates, and co-transfected with wild-type or mutant luciferase reporters and the miR-422a mimic or miR-NC using the X-tremeGENE transfection reagent (Roche Diagnostics). To evaluate the $\beta$-catenin/CF-4 transcriptional activity, TOP-FLASH and FOP-FLASH luciferase reporter constructs were used. TOP-FLASH (with repeats of the TCF binding site) or FOP-FLASH (with repeats of the mutant TCF binding site) plasmids (EMD Millipore) were transfected into LN229 and U87 cells transfected with miR-422a mimic. Furthermore, RPN2 overexpression plasmid transfection was performed $24 \mathrm{~h}$ after miRNA transfection. Following a further $48 \mathrm{~h}$ incubation, luciferase activity was measured using the Dual-Luciferase Reporter assay system (Promega Corporation), and Renilla luciferase activity was used as an internal control.
RNA immunoprecipitation (RIP) assay. The Magna RIP RNA-Binding Protein Immunoprecipitation kit (EMD Millipore) was used to perform RIP assays according to the manufacturer's instructions. LN229 and U87 cells transfected with miR-422 mimic or miR-NC for $48 \mathrm{~h}$ were collected by centrifugation at $150 \mathrm{xg}$ for $5 \mathrm{~min}$ at $4^{\circ} \mathrm{C}$ and incubated overnight at $4^{\circ} \mathrm{C}$ with RIP buffer containing protein $\mathrm{A} / \mathrm{G}$ magnetic beads coated with anti-Ago 2 or anti-IgG antibody (catalog no. 03-110; EMD Millipore; 1:5,000) as a negative control. Following overnight incubation at overnight at $4^{\circ} \mathrm{C}$ with rotation, and following washing, RNase-free DNase I and proteinase K (EMD Millipore) were used to purify the RNA in the immunoprecipitated complex, and RT-qPCR was then performed to detect the RPN2 expression level, as described previously.

Viability assay. Cell viability was analyzed by Cell Counting Kit-8 (CCK-8; Dojindo Molecular Technologies, Inc.) according to the manufacturer's instructions. Following transfection with miR-422a mimic or co-transfection with miR-422a mimic and RPN2 plasmid, LN229 and U87 cells were incubated for $24,48,72$ and $96 \mathrm{~h}$. CCK-8 solution $(10 \mu \mathrm{l})$ was then added to each well, and the absorbance at $490 \mathrm{~nm}$ was measured after incubation for $2 \mathrm{~h}$ to estimate the number of viable cells.

Cell cycle analysis. Cell cycle analysis was performed by flow cytometry, and transfected and control LN229 and U87 cells in the log phase of growth were harvested by trypsinization, washed with PBS, fixed with $75 \%$ ethanol overnight at $4^{\circ} \mathrm{C}$ and then incubated with RNase at $37^{\circ} \mathrm{C}$ for $30 \mathrm{~min}$. A total of $1 \times 10^{4}$ nuclei were assessed with a FACS Calibur Flow Cytometer (Becton, Dickinson and Company), and DNA histograms were analyzed using Modfit software (Modfit LT 4.0; Becton, Dickinson and Company). Experiments were performed in triplicate.

Transwell assay. Transwell membranes coated with Matrigel (BD Biosciences) were placed in an incubator for $30 \mathrm{~min}$ at $37^{\circ} \mathrm{C}$, which were used to quantify glioma cell invasion. Transfected cells were plated at $5 \times 10^{4}$ per well in the upper chamber in serum-free medium. DMEM supplemented with $10 \%$ FBS $(600 \mu \mathrm{l})$ were used as a chemoattractant and placed in the bottom chamber. After $24 \mathrm{~h}$ of incubation, the filters were gently removed, and the medium was removed from the upper chamber. The cells that had migrated through the Matrigel into the pores of the inserted filter were fixed with $100 \%$ methanol, stained with hematoxylin for $20 \mathrm{~min}$ at room temperature, and mounted. The number of cells that invaded through the Matrigel was counted in three randomly selected visual fields from the central and peripheral portions of the filter using an inverted microscope (magnification, x200).

Apoptosis assay. Apoptosis was quantified following transfection by assessing Annexin V labelling and caspase 3/7 activity. For the Annexin V assay, an annexin V-FITC-labeled apoptosis detection kit (Abcam) was used according to the manufacturer's protocol. Caspase $3 / 7$ activity was analyzed using Caspase-Glo 3/7 reagent (catalog no. G8091; Promega Corporation). Briefly, caspase-Glo $3 / 7$ reagent (100 $\mu \mathrm{l}$ ) was 
added to a white-walled 96-well plate, which was gently mixed using a plate shaker at $50 \mathrm{x}$ g for $30 \mathrm{sec}$. Following incubation at room temperature for 1-2 $\mathrm{h}$, the luminescence of each sample was measured in a plate-reading luminometer.

Western blotting. The total protein extraction of different transfected LN229 and U87 cells was performed using ExKine Total Protein Extraction kit (Abbkine), and nuclear protein extraction was performed using DUALXtract Cytoplasmic and Nuclear Protein Extraction kit (Dualsystems Biotech AG), according to manufacturer's protocol. Protein concentrations were determined using a BCA Protein assay kit (Thermo Fisher Scientific, Inc.). Equal amounts of protein (30 $\mu \mathrm{g} /$ lane) were separated by $10 \%$ SDS-PAGE and subsequently transferred to PVDF membranes (EMD Millipore). After blocking in 5\% skimmed milk for $1 \mathrm{~h}$, the membranes were incubated with diluted primary antibodies at $4^{\circ} \mathrm{C}$ overnight. After washing with TBST (1\% Tween-20), the membranes were incubated with horseradish peroxidase-conjugated secondary antibodies (1:5,000; cat. nos. ab222772 and ab222759; Abcam) for $1 \mathrm{~h}$ at room temperature, and the immune-reactive bands were visualized using ECL Western Blot Detection reagents (EMD Millipore). The expression levels were normalized to the levels of $\beta$-actin or GAPDH of the total protein and fibrillarin of nuclear protein. The protein band intensities were quantified using ImageJ software (version 1.48; National Institutes of Health). The primary antibodies used were as follows: RPN2 (1:200; catalog no. ab244399; Abcam), $\beta$-catenin (1:5,000; catalog no. ab32572; Abcam), cyclinD1 (1:10,000; catalog no. ab134175; Abcam), TCF4 (1:10,000; catalog no. ab76151; Abcam), c-myc (1:1,000; ab39688; Abcam), $\beta$-actin (1:5,000; catalog no. ab6276; Abcam), fibrillarin (1:5,000; catalog no. ab4566; Abcam) and GAPDH (1:1,000; catalog no. 5174; Cell Signaling Technology, Inc.).

Immunohistochemistry (IHC) analysis. For IHC assays, detailed protocols were performed as previously described (25). Briefly, the animal tumor samples were fixed with $10 \%$ formalin for $48 \mathrm{~h}$ at room temperature, embedded with paraffin, and sliced into $4-\mu \mathrm{m}$ sections, followed by dewaxing. Subsequently, these sections were incubated with $3 \%$ hydrogen peroxide for 20 min to block endogenous peroxidase. Following antigen retrieval, the slides were blocked with $10 \%$ normal goat serum (Beijing Solarbio Science \& Technology Co., Ltd.) for $30 \mathrm{~min}$ and then incubated at $4{ }^{\circ} \mathrm{C}$ overnight with appropriate primary antibody. After washing with PBS, the slides were incubated with biotinylated secondary antibody (cat. no. Sp-9001; 1:1,000; OriGene Technologies, Inc.) for $1 \mathrm{~h}$ at room temperature. A DAB Horseradish Peroxidase Color Development kit (Beyotime Institute of Biotechnology) was used for color development, and neutral gum (Sinopharm Chemical Reagent Co., Ltd.) was used for sealing. Subsequently, five fields were randomly selected and imaged using the Olympus X71 inverted microscope (Olympus Corporation). The images were assessed using Image-Pro Plus 6.0 (Media Cybernetics, Inc.). The antibodies used for IHC were as follows: RPN2 (1:200; cat. no. ab244399; Abcam), $\beta$-catenin (1:500; cat. no. ab32572; Abcam), c-myc (1:100; cat. no. ab39688; Abcam), caspase 3 (1:100; cat. no. 9662; Cell Signaling Technology, Inc.) and PCNA (1:4,000; cat. no. 2586; Cell Signaling Technology, Inc.).
Xenograft model with nude mice. All animal protocols were performed with the approval of the Animal Care and Used Committee of Tianjin Huanhu Hospital (Tianjin, China). A total of 10 female BALB/c nude mice at 5 weeks of age (weight, 18-21 g) were purchased from the Animal Center of the Cancer Institute, Chinese Academy of Medical Science. Animal welfare was taken seriously and all the mice were housed with filtered air, a 12-h light/dark cycle, regulated temperature $\left(25 \pm 2^{\circ} \mathrm{C}\right)$ and humidity $(50 \pm 10 \%)$, and with ad libitum access to sterilized food and water. A total of $1 \times 10^{7}$ U87 GBM cells in $100 \mu \mathrm{l}$ PBS were subcutaneously injected into the left flank region of nude mice. When the tumor volume reached $100 \mathrm{~mm}^{3}$, mice were randomly divided into two groups (5 mice per group) and subsequently treated with oligonucleotides (10 $\mu \mathrm{g}$ miR-422a mimic or miR-NC) with a mixture of $10 \mu \mathrm{l}$ Lipofectamine 2000 (Invitrogen; Thermo Fisher Scientific, Inc.) through local injection of the xenograft tumor at multiple sites, respectively. The treatment was performed once every 3 days for 27 days. The tumor volume was monitored with a caliper, using the formula: Volume=length $\mathrm{x}$ width ${ }^{2} / 2$. The health and behavior of mice were monitored daily. Humane endpoints used in the study to verify when to euthanize the mice included: i) Tumors, $2.0 \mathrm{~cm}$ diameter; ii) weight loss, $\geq 20 \%$; iii) tumor ulceration, iv) abnormal posture; and v) quick weight loss assessed by observations and weighing. At the termination of the experiment, no mice reached these criteria. The mice were anesthetized by injecting $1 \%$ pentobarbital sodium (100 mg/kg body weight) and sacrificed by cervical vertebra dislocation, when the diameter of the largest tumor was $1.93 \mathrm{~cm}$. If there was no heartbeat for $30 \mathrm{sec}$ accompanied by no response to the toe pinch reflex, mice were considered to be euthanized. Finally, tumor tissues were removed, tumor weight was calculated, and RNA extraction and IHC assays were performed.

Statistical analysis. All statistical analyses were performed using GraphPad software version 6.0 (GraphPad Software, Inc.) or SPSS 23.0 (IBM Corp.). Data are presented as the mean \pm standard deviation of at least three independent experiments. An unpaired Student's t-test and one-way ANOVA were performed to analyze significant differences between two groups or multiple groups, respectively. Additionally, Tukey's test was used for multiple comparisons following ANOVA. Fisher's exact test was used to analyze the associations between miR-422a and clinicopathological characteristics of patients with glioma. The correlation between miR-422a and c-myc expression in 29 GBM patients was assessed using Pearson's correlation analysis. The survival curves from online databases were plotted using the Kaplan-Meier method and compared using the log-rank test. $\mathrm{P}<0.05$ was considered to indicate a statically significant difference.

\section{Results}

miR-422a is significantly downregulated in GBM specimens and cell lines. To investigate the expression level of miR-422a in glioma cell lines and samples, total RNA was isolated from eight glioma cell lines, GBM specimens and normal brain tissues, and the levels of miR-422a were evaluated by RT-qPCR. The results demonstrated that miR-422a 

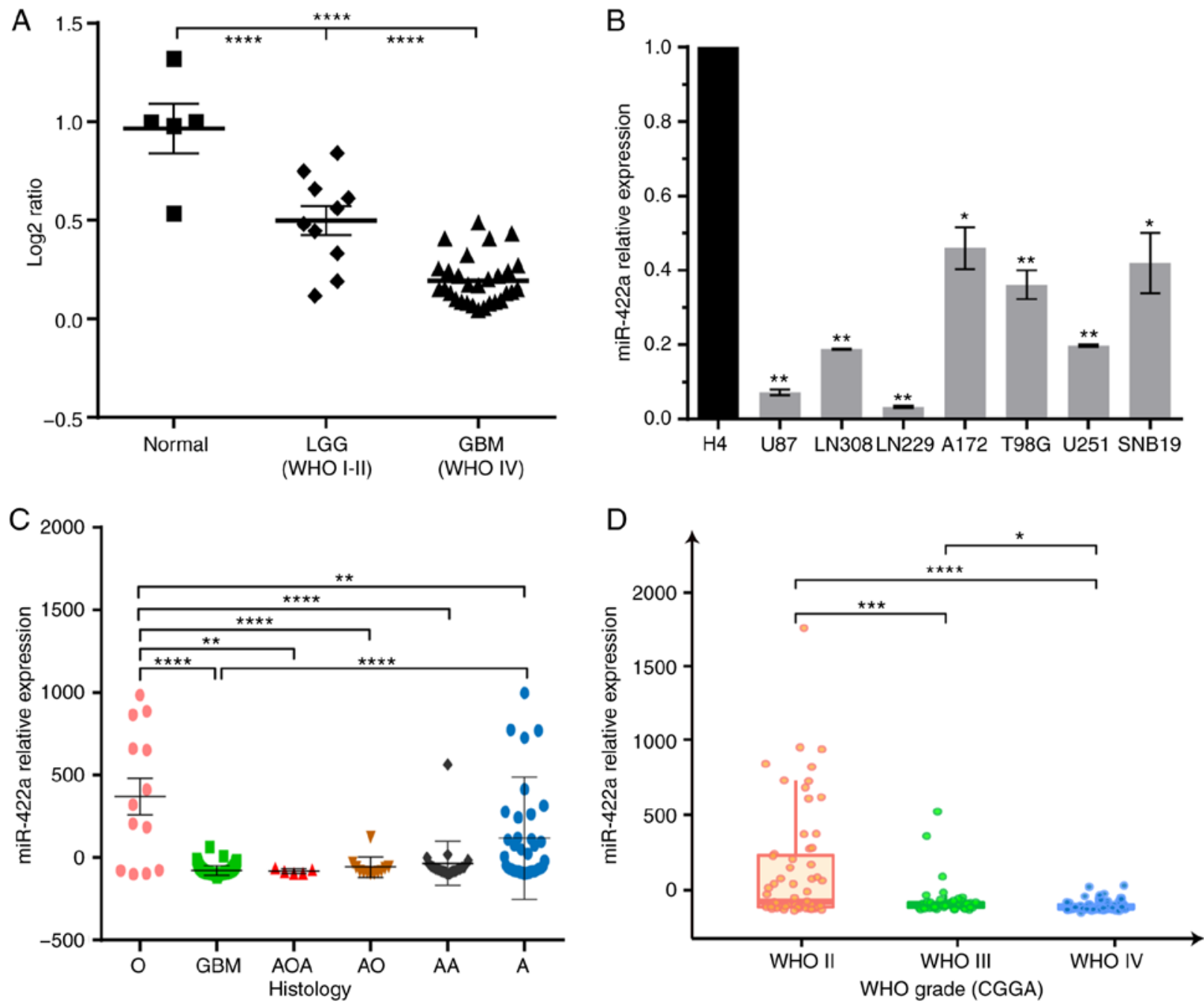

Figure 1. miR-422a is significantly downregulated in GBM clinical samples and cell lines. (A) RT-qPCR was used to evaluate the miR-422a expression in 10 LGG (WHO grade I-II) and 29 GBM (WHO grade IV) resected specimens compared with 5 normal brain tissues. (B) RT-qPCR analysis of the low-grade H4 cell line and GBM cell lines (U87, LN308, LN229, A172, T98G, U251 and SNB19). "P $<0.05,{ }^{* *} \mathrm{P}<0.01$ vs. H4 cells. (C) The association between miR-422a and histology in gliomas according to the CGGA database. (D) miR-422a expression was negatively associated with the WHO grade of glioma samples from the CGGA database. ${ }^{*} \mathrm{P}<0.05,{ }^{* *} \mathrm{P}<0.01,{ }^{* * *} \mathrm{P}<0.001,{ }^{* * * *} \mathrm{P}<0.0001$. miR-422a, microRNA-422a; GBM, glioblastoma multiforme; RT-qPCR, reverse transcription-quantitative PCR; WHO, World Health Organization; CGGA, Chinese Glioma Genome Atlas; LGG, low-grade glioma.

was significantly downregulated in glioma tissues compared with normal brain tissues, and miR-422a expression was significantly lower in WHO grade IV GBM compared with in low-grade glioma samples (WHO grades I-II) (Fig. 1A). The clinicopathological characteristics of 39 glioma specimens are summarized in Table I, and no significant associations were identified between miR-422a expression and other clinicopathological variables, including gender and age, although it was significantly association with histology. Additionally, miR-422a was also significantly lower in the seven GBM cell lines compared with the low-grade cell line H4 (Fig. 1B). Furthermore, miR-422a expression was analyzed in CGGA, which revealed that the miR-422a expression level was associated with the histopathological subtypes, specifically, the expression in subtypes $\mathrm{O}$ and $\mathrm{A}$ (WHO II) were significantly higher compared with in the subtypes of AOA, AO and AA (WHO III) and GBM (WHO IV) (Fig. 1C). Similarly, a high expression of miR-422a was significantly associated with a lower WHO grade (Fig. 1D). These data indicate that miR-422a may act as a key tumor suppressor in glioma progression.
Ectopic miR-422a expression suppresses the GBM malignant phenotype. Based on the low expression of miR-422a in GBM, the present study then investigated how the malignant phenotype of GBM was mediated by miR-422a. LN229 and U87 cells were transfected with miR-422a mimic or miR-NC, and RT-qPCR was performed to verify miR-422a overexpression (Fig. 2A). The CCK-8 assay revealed a significant decrease in cell viability for miR-422a mimic-transfected GBM cells compared with the miR-NC group (Fig. 2B). Flow cytometry analysis demonstrated a significant increase in G1 cell cycle arrest in miR-422a mimic-transfected LN229 and U87 cells (Fig. 2C). Additionally, in vitro Annexin-V and Transwell assays revealed that miR-422a overexpression significantly enhanced tumor cell apoptosis and significantly reduced the number of invasive LN229 and U87 cells (Fig. 3D and E). These results suggest that $\mathrm{miR}-422 \mathrm{a}$ overexpression suppressed GBM cell proliferation and invasion, and promoted G1 cell cycle arrest and apoptosis, indicating a crucial role in the progression of GBM. 
Table I. Associations between miR-422a and clinicopathological characteristics of patients with glioma.

\begin{tabular}{|c|c|c|c|c|}
\hline \multirow[b]{2}{*}{ Variable } & \multirow[b]{2}{*}{ No. of cases } & \multicolumn{2}{|c|}{$\begin{array}{l}\text { miR-422a } \\
\text { expression }\end{array}$} & \multirow[b]{2}{*}{ P-value } \\
\hline & & Low, n & High n & \\
\hline Sex & & & & 0.1532 \\
\hline Female & 15 & 7 & 8 & \\
\hline Male & 24 & 5 & 19 & \\
\hline Age, years & & & & $>0.999$ \\
\hline$<42$ & 20 & 13 & 7 & \\
\hline$\geq 42$ & 19 & 13 & 6 & \\
\hline Histology & & & & 0.0015 \\
\hline Astrocytoma & 10 & 2 & 8 & \\
\hline Glioblastoma & 29 & 23 & 6 & \\
\hline
\end{tabular}

RPN2 is a direct target of miR-422a. To verify the downstream effectors of miR-422a, PITA, miRmap and miRanda databases were used. Based on these databases, it was identified that the 'seed sequence' of miR-422a matched the 3'UTR of the RPN2 mRNA (Fig. 3A). To determine whether RPN2 expression was modulated by miR-422a, western blotting was performed to detect RPN2 protein expression in LN229 and U87 cells transfected with miR-422a mimic, and the results revealed that miR-422a overexpression significantly reduced the RPN2 expression level (Fig. 3B). To confirm that RPN2 was a direct target of miR-422a, luciferase reporter constructs carrying the RPN2 3'UTR with wild-type or mutant miR-422a binding sites were constructed and co-transfected with miR-422a mimic and miR-NC. In comparison to miR-NC, miR-422a mimic significantly decreased the luciferase activities (Fig. 3C). However, the miR-NC and miR-422a mimic did not affect the luciferase activity in mutant constructs. Furthermore, a RIP assay was performed using an Ago 2 antibody to investigate the direct association between miR-422a and RPN2. The results indicated that RPN2 was enriched in Ago2-coated beads compared with the IgG control group and that miR-422a mimic resulted in a marked upregulation of RPN2 level in the Ago2 immunoprecipitation complex in LN229 and U87 cells (Fig. 3D). In addition, RPN2 expression level was detected in five normal and 29 GBM specimens, and RPN2 was significantly upregulated in the GBM samples compared with the normal samples (Fig. 3E). These data indicate that miR-422a directly regulates RPN2 expression by binding to the 3'UTR of RPN2.

miR-422 a suppresses the Wnt/ $\beta$-catenin signaling pathway, at least partially through RPN2. Our previous studies confirmed that RPN2 promotes activation of the $\mathrm{Wnt} / \beta$-catenin signaling pathway (data not shown). In combination with the fact that RPN2 is a direct functional target of miR-422a, the present study further investigated whether miR-422a also regulates the Wnt/ $\beta$-catenin signaling pathway via RPN2. RT-qPCR demonstrated successful overexpression of RPN2 in LN229 and $\mathrm{U} 87$ cells following transfection with RPN2 overexpression plasmid (Fig. 4A), and results of the western blot analysis of RPN2 expression in LN229 and U87 cells transfected with miR-422a mimic or miR-422a and RPN2 overexpression plasmid are shown in Fig. 4B, indicating that the transfection of RPN2 overexpression plasmid can markedly recover RPN2 protein level in LN229 and U87 cells transfected with miR-422a mimic.

A TOP/FOP assay was used to analyze regulation of $\beta$-catenin/TCF4 transcriptional activity by miR-422a, and the results demonstrated that miR-422a significantly suppressed TOP luciferase activity, with no apparent change in FOP activity (Fig. 4C). Furthermore, ectopic miR-422a expression could inhibit TCF4, c-myc and cyclinD1 expression, and $\beta$-catenin nuclear translocation, as verified by RT-qPCR and western blotting (Fig. 4D-F). However, overexpression of RPN2 following the transfection with miR-422a reversed the effects mediated by miR-422a (Fig. 4C-F). To further verify the relationship between miR-422a and Wnt signaling, c-myc, a critical downstream factor of the $\mathrm{Wnt} / \beta$-catenin pathway, was detected by RT-qPCR, which revealed that it was significantly higher in GBM tissue samples compared with normal samples (Fig. 4G). Additionally, Pearson's correlation analysis demonstrated that miR-422a was negatively correlated with the expression of c-myc in 29 GBM patient samples (Fig. 4H). These data demonstrate that miR-422a can attenuate the Wnt/ $\beta$-catenin signaling pathway, at least partially through RPN2 in LN229 and U87 cells.

$R P N 2$ is required for the biological effects of $m i R-422 a$ on the GBM malignant phenotype. To further verify the functional association between miR-422a and its target RPN2, the role of RPN2 in the miR-422a-mediated effect on the proliferation, invasion and apoptosis of GBM cells was investigated. When RPN2 was ectopically overexpressed in LN229 and U87 cells transfected with miR-422a mimic, the inhibition of proliferation and invasion, and the promotion of apoptosis induced by miR-422a was partially reversed (Fig. 5A-D). Furthermore, the knockdown of RPN2 by shRPN2 significantly enhanced the caspase-3/7 activity (Fig. 5E), which was consistent with the effect mediated by miR-422a overexpression. These results indicate that miR-422 inhibits the GBM malignant phenotype, partially through the oncogene RPN2.

miR-422a inhibits tumor growth in vivo. Based on the in vitro experimental findings, the effects of miR-422a on tumor growth and $\mathrm{Wnt} / \beta$-catenin signaling were further examined in vivo. U87 cells were implanted into the left flanks of nude mice by subcutaneous injection. miR-422a mimic and miR-NC were injected in multiple sites of the tumor every 3 days. The study was terminated on day 27 , and the tumors were excised and further analyzed. The tumor growth curve was generated from data obtained every 3 days, which demonstrated that the overexpression of miR-422a significantly reduced the tumor volume (Fig. 6A). Additionally, a significant decrease in tumor weight was observed for the miR-422a mimic-treated tumors compared with the miR-NC-treated tumors (Fig. 6B). Evidence that miR-422a mimic significantly increased miR-422a expression in tumor tissues is presented in Fig. 6C. Furthermore, the IHC assay demonstrated that miR-422a overexpression significantly inhibited RPN2, $\beta$-catenin, c-myc 

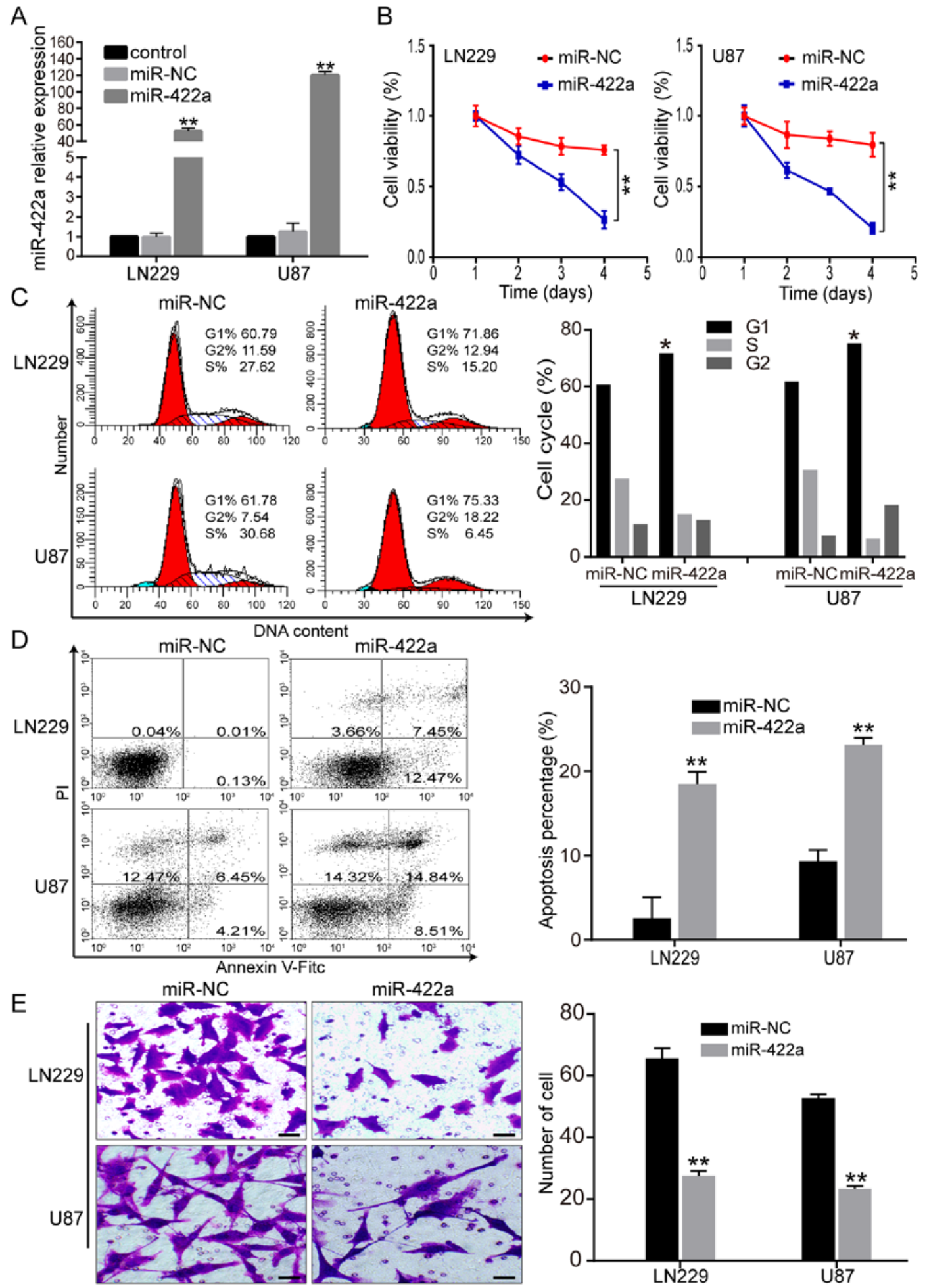

Figure 2. Ectopic miR-422a expression suppresses GBM growth in vitro. (A) Reverse transcription-quantitative PCR was performed to confirm that miR-422a was significantly overexpressed following transfection with miR-422a mimic compared with miR-NC. (B) CCK8 cell viability assay of LN229 and U87 cells following miR-422a overexpression. (C) Cell cycle distribution analysis was performed by flow cytometry. (D) Apoptosis detection was performed by Annexin V-PI staining. (E) Transwell assays were performed to examine the LN229 and U87 invasive ability after transfection with miR-422a overexpression plasmid (magnification, $\mathrm{x} 200$ ). ${ }^{*} \mathrm{P}<0.05,{ }^{* *} \mathrm{P}<0.01$ vs. miR-NC. miR-422a, microRNA-422a; GBM, glioblastoma multiforme; NC, negative control; PI, propidium iodide.

and PCNA expression, and increased the caspase 3 expression level (Fig. 6D), which was consistent with the in vitro results.

Increased RPN2 expression is associated with poor prognosis in human glioma. Based on the association between miR-422a and RPN2, RPN2 expression and its correlation with prognosis in the CGGA dataset (CGGA Mseq325) and the UALCAN and GEPIA public databases was investigated. The CGGA data revealed that the mRNA level of RPN2 was significantly higher in high-grade gliomas compared with the low-grade glioma (Fig. 7A). Retrospective analysis of the clinical outcome of 

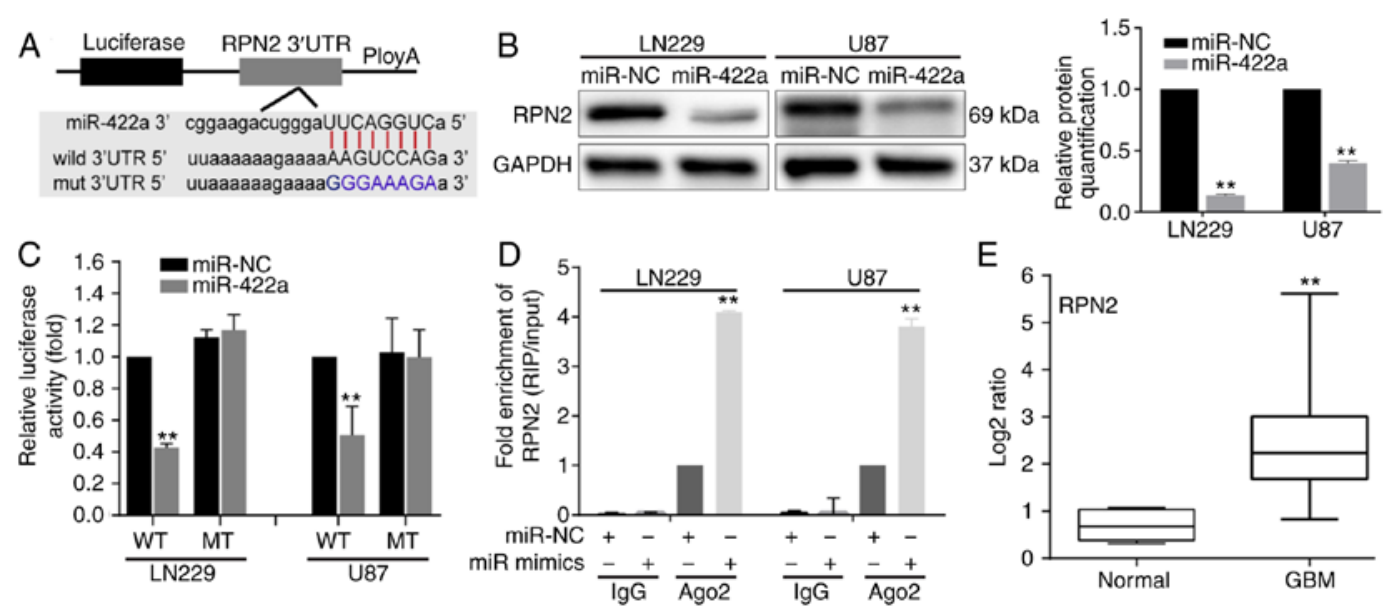

Figure 3. RPN2 is a direct target of miR-422a. (A) A schematic diagram of the seed sequence of miR-422a that matches the RPN2 3'UTR and the design of wild-type or mutant RPN2 3'UTR constructs including reporters. (B) Changes in RPN2 expression in LN229 and U87 cells transfected with miR-NC or miR-422a mimic were detected by western blotting. GAPDH was used as a loading control. The relative protein quantification was performed with ImageJ software. ${ }^{* *} \mathrm{P}<0.01$ vs. miR-NC. (C) Luciferase reporter assays with LN229 and U87 cells following co-transfection with the WT or MT RPN2 3'UTR of RPN2 and miR-422a mimic or miR-NC. ${ }^{* *} \mathrm{P}<0.01 \mathrm{vs}$. miR-NC. (D) A RIP assay was conducted to detect the enrichment level of RPN2 in IgG or Ago2 immunoprecipitation complexes in LN229 and U87 cells transfected with miR-422a or miR-NC. ${ }^{* *} \mathrm{P}<0.01$ vs. miR-NC. (E) RPN2 expression levels in five normal controls and 29 GBM patients. ${ }^{* *} \mathrm{P}<0.01$ vs. Normal. RPN2, ribophorin II; miR-422a, microRNA-422a; 3'UTR, 3'-untranslated region; NC, negative control; WT, wild-type; MT, mutant; RIP, RNA immunoprecipitation.
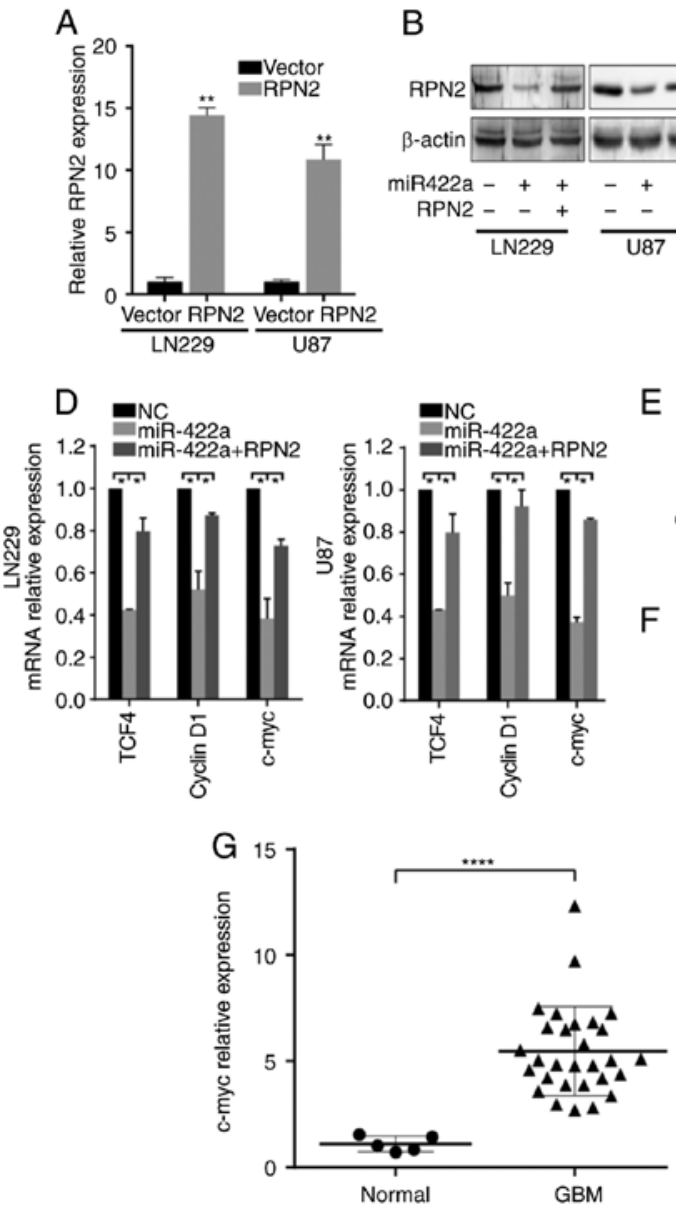

E
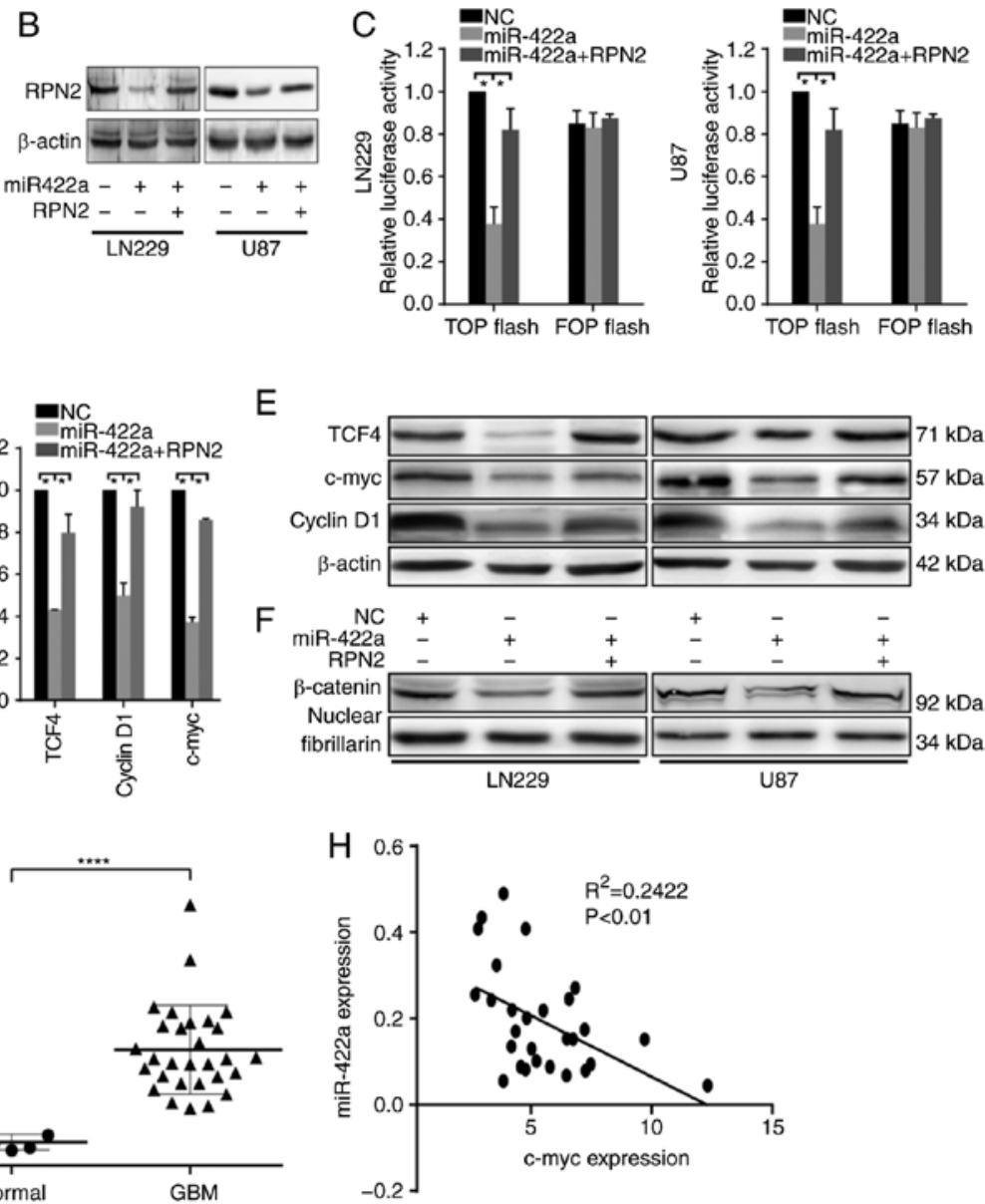

Figure 4. Ectopic miR-422a inhibits $\beta$-catenin/TCF4 transcriptional activity, and RPN2 restoration in miR-422a-overexpressing LN229 and U87 cells can partially reverse this effect. (A) RT-qPCR analysis of the transfection efficiency of RPN2 overexpression plasmid. (B) Western blot analysis of RPN2 expression in LN229 and U87 cells following transfection with miR-422a mimic or co-transfection with miR-422a and RPN2 overexpression plasmid. ${ }^{* *} \mathrm{P}<0.01$ vs. Vector. (C) LN229 and U87 cells were co-transfected with TOP/FOP, miR-422a mimic or RPN2-overexpression plasmid, and analyzed after 48 h by luciferase reporter assay. ${ }^{*} \mathrm{P}<0.05$ vs. NC. (D) RT-qPCR and (E) western blotting were performed to examine TCF4, c-myc and cyclinD1 expression after transfection with miR-422a mimic or co-transfection with miR-422a and RPN2. ${ }^{*} \mathrm{P}<0.05$ vs. NC. (F) Western blotting of $\beta$-catenin in the nucleus. (G) RT-qPCR analysis of the Wnt/ $\beta$-catenin pathway-related protein c-myc in 29 patients with GBM. ${ }^{* * * *} \mathrm{P}<0.0001$. (H) A significant inverse correlation was identified between miR-422a and c-myc expression in GBM specimens. RPN2, ribophorin II; miR-422a, microRNA-422a; NC, negative control; GBM, glioblastoma multiforme. 

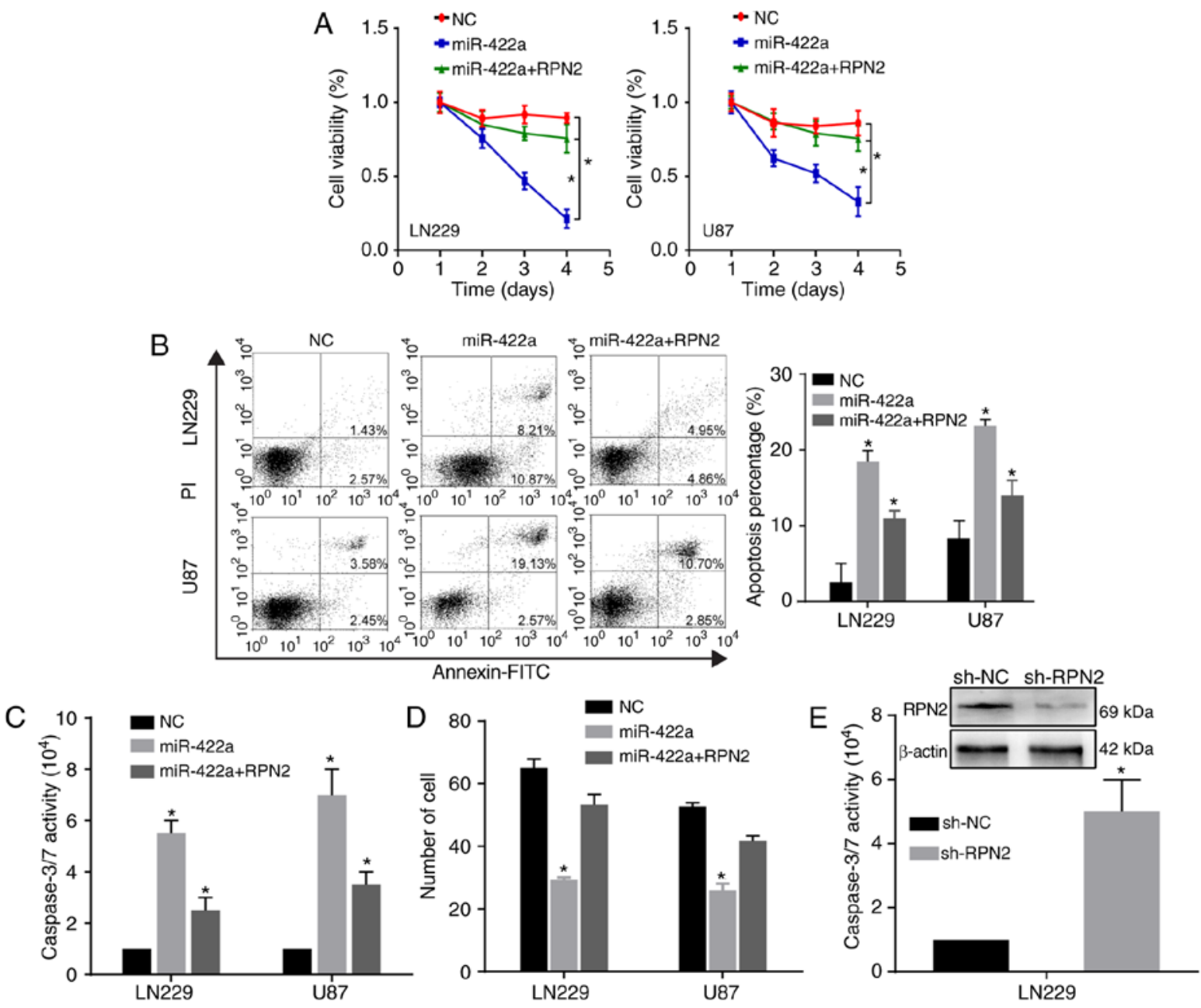

Figure 5. RPN2 restoration in miR-422a-overexpressing LN229 and U87 cells reverses the inhibitory effects of miR-422a on glioblastoma multiforme proliferation and invasion. (A) Following miR-422a transfection for $24 \mathrm{~h}$, cells were transfected with RPN2 overexpression plasmid. After $72 \mathrm{~h}$, a CCK8 assay was used to detect the RPN2-mediated effect on proliferation by miR-422a. ${ }^{*} \mathrm{P}<0.05$. (B) Annexin V-PI and (C) caspase-3/7 activity assays were performed to detect the RPN2-mediated effect of miR-422a on apoptosis. "P<0.05 vs. miR-NC. (D) A Transwell assay was performed to evaluate the RPN2-mediated effect of miR-422a on the invasion of LN229 and U87 cells. "P<0.05 vs. miR-NC. (E) Caspase 3/7 activity assay following knockdown of RPN2 in LN229 cells and the identification of RPN2 downregulation by western blotting. "P<0.05 vs. sh-NC. RPN2, ribophorin II; miR-422a, microRNA-422a; NC, negative control; PI, propidium iodide; sh, short hairpin RNA.

these patients revealed that low expression of RPN2 was significantly associated with a longer overall survival, in both primary and recurrent gliomas (Fig. 7B and C). Further analysis of RPN2 expression and relevant clinical characteristics indicated that RPN2 expression was significantly associated with IDH1 gene mutation status, age and $1 p / 19 q$ codeletion status (Fig. S1A-C). Furthermore, the low-grade glioma data analysis of the UALCAN database demonstrated that RPN2 expression in grade 3 was significantly higher than that in grade 2 (Fig. 7D), and the higher RPN2 expression was significantly associated with a poorer survival (Fig. 7E). Moreover, the data obtained from the 'Survival Map' module of GEPIA demonstrated that the high RPN2 expression group had a poorer overall survival and disease free survival compared with the low RPN2 expression group of patients with low-grade glioma $(\mathrm{n}=514)$ and gliomas, including low-grade and high-grade glioma ( $\mathrm{n}=676$ ) (Fig. 7F-I). Hence, these data indicate that RPN2 predicts poor prognosis and is associated with the progression of gliomas.

\section{Discussion}

Accumulating data have demonstrated that miR-422a, which serves as a key anticancer gene, exerts a crucial influence on the initiation, development and drug resistance of several tumors. He et al (26) reported that miR-422a downregulation contributes to malignancy by targeting pyruvate dehydrogenase kinase 2 in gastric cancer. Zhang et al (9) demonstrated that miR-422a suppresses tumor growth and metastasis in hepatocellular carcinoma. Additionally, miR-422a inhibits osteosarcoma cell cycle arrest and induces apoptosis by directly targeting BCL2L2 and KRAS (27). Furthermore, a study of glioma analyzing a TCGA dataset indicated that miR-422a expression predicts the neural subtype of GBM and patient outcome (28). In combination with the public database analysis, this evidence prompted us to further explore the expression of miR-422a in clinical tissues of GBM with different grades and GBM cell lines. Moreover, the role of miR-422a in the malignant phenotype of GBM and the underlying antitumor mechanism was also investigated. 

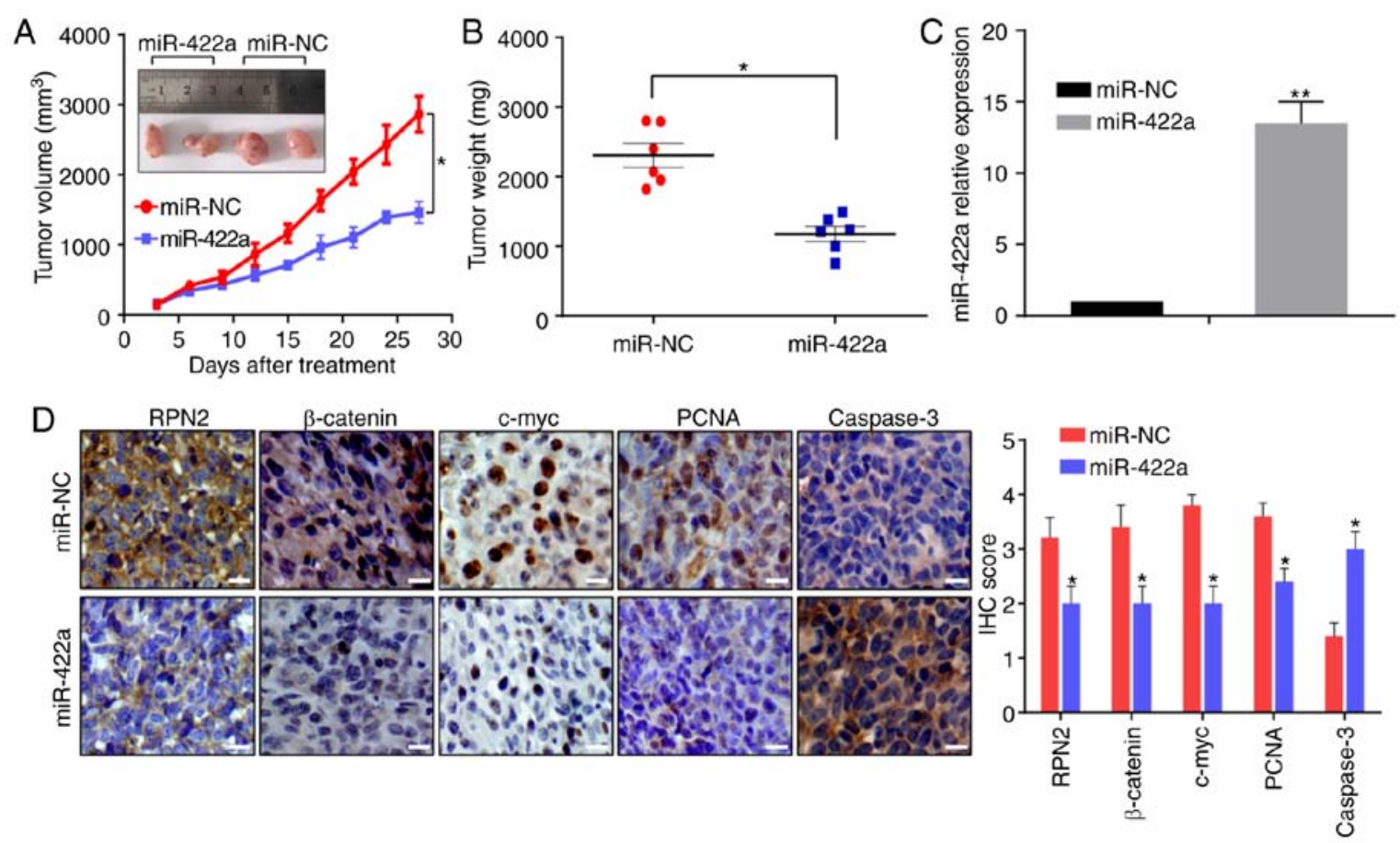

Figure 6. miR-422a inhibits GBM xenograft tumor growth. (A) U87 cells were subcutaneously injected into nude mice. When tumors were established uniformly in the two groups, miR-422a was administered by multisite injection every 3 days. Tumor volumes were evaluated every 3 days during treatment. ${ }^{*} \mathrm{P}<0.05$. (B) At the termination of the experiment, tumor weights were measured. ${ }^{*} \mathrm{P}<0.05$. (C) Reverse transcription-quantitative PCR was performed to examine miR-422a expression in resected tumor tissues. ${ }^{* *} \mathrm{P}<0.01$ vs. miR-NC. (D) IHC assays were performed to assess RPN2, $\beta$-catenin, c-myc, PCNA and caspase3 expression in xenograft tumor sections. Scale bar, $50 \mu \mathrm{m}$. "P<0.05 vs. miR-NC. IHC, immunohistochemistry; miR-422a, microRNA-422a; $\mathrm{NC}$, negative control; RPN2, ribophorin II.

Although previous studies revealed that miR-422a markedly suppressed glioma cell proliferation, migration and invasion by targeting PIK3CA, insulin-like growth factor 1 (IGF1) and IGF1 receptor (IGF1R), which are new prognostic biomarkers for human glioblastoma $(14,29,30)$, the detailed mechanism of the miR-422a-mediated inhibitory effect on tumor growth remains poorly understood. The present study first confirmed the downregulation of miR-422a, which was negatively associated with the WHO grade, and identified its antitumor function. Mechanistically, by analyzing PITA, miRmap and miRdanda public databases, it was identified that RPN2 had highly conserved binding sites for miR-422a in its 3'UTR region. Experimental evidence from a luciferase reporter assay, western blotting and RIP assay verified that RPN2 was a direct target of miR-422a. These data also demonstrated that miR-422a suppressed GBM growth at least partially through RPN2. Furthermore, the present study investigated the role of RPN2 in the miR-422a-mediated regulation of GBM biological behaviors and Wnt/ $\beta$-catenin signaling pathway. Collectively, the findings revealed a novel $\mathrm{miR}-422 \mathrm{a} / \mathrm{RPN} 2 / \mathrm{Wnt} / \beta$-catenin axis implicated in GBM development and progression, deepening the understanding of the etiology of glioma.

Extensive studies have verified that RPN2 upregulation is involved in the progression of various malignancies, and that RPN2-mediated glycosylation of P-gp (encoded by MDR1) is responsible for drug resistance in a number of cancers, including breast cancer, ovarian cancer, gastric cancer and esophageal squamous cell carcinoma (31-34). Additionally, it has been reported that the P-gp gene is closely associated with poor prognosis and temozolomide (TMZ) resistance in high-grade gliomas $(35,36)$. Accordingly, whether miR-422a modulates TMZ chemosensitivity via RPN2 may be worth investigating. However, to the best of our knowledge, there have been no reports regarding the expression and function of RPN2 in gliomas. Our previous unpublished study systematically evaluated RPN2 upregulation in glioma specimens and GBM cell lines and investigated the tumor-promoting mechanism (data not shown). Furthermore, in the present study, survival analysis from online public databases indicated that high RPN2 expression is associated with poor prognosis. A limitation of the current study is that a log-rank test may not applicable for survival plots where late-stage crossover is present, although it is the most common way to analyze survival differences. However, the survival time of gliomas is generally similar to the normal distribution, few patients have a very short or long survival time. Hence, the late-stage crossover observed for patients with very long survival may not be significant. Indeed, further verification of these data is necessary to determine the prognostic significance of RPN2 using a weighted test, such as Renyi or Cramér-von Mises.

Wnt $/ \beta$-catenin signaling is one of the most important oncogenic pathways in GBM and represents a promising target for glioma treatment $(37,38)$. $\beta$-catenin in the nucleus interacts with the TCF/LEF family to regulate the transcription of multiple genes, including c-myc and cyclinD1, while GSK-3 $\beta$ promotes its degradation contributing to Wnt signal inactivation (37-39). Takahashi et al (40) confirmed that RPN2 antagonizes GSK3 $\beta$ through physical interactions and subsequently suppresses heat shock protein-containing HSP70 and 
A
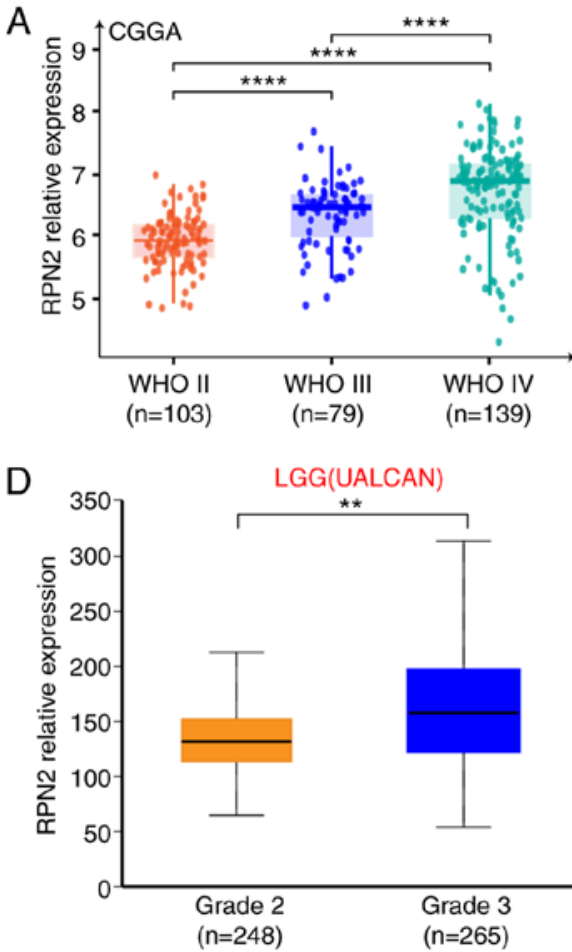

G

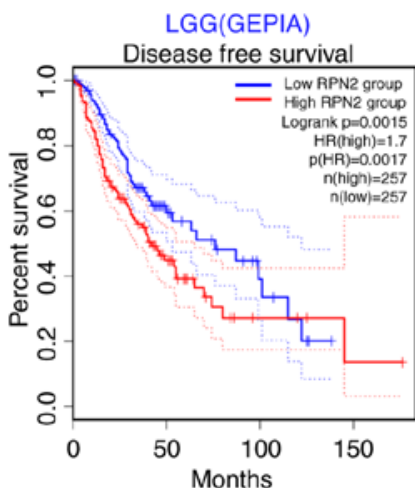

B

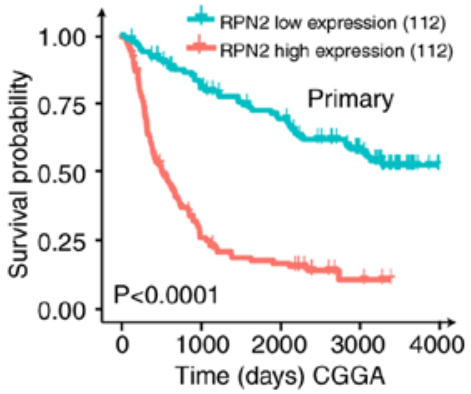

E

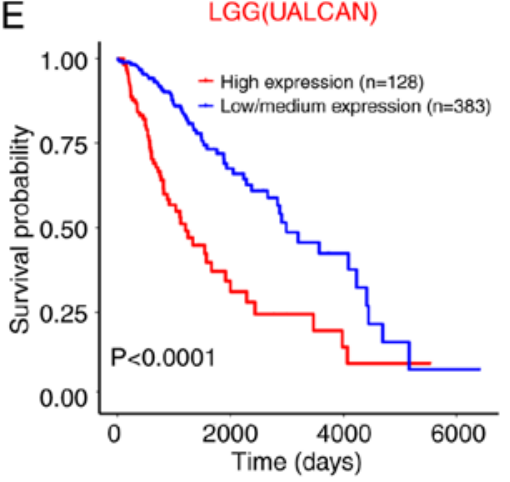

$\mathrm{H}$

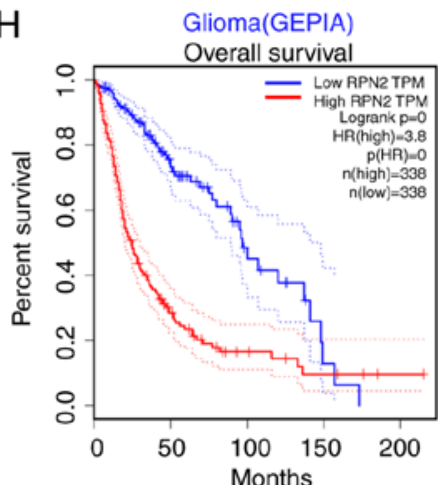

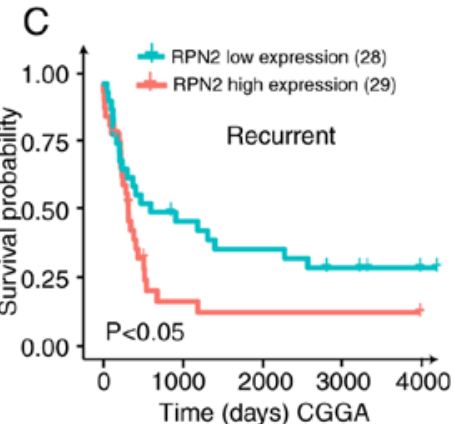

$\mathrm{F}$

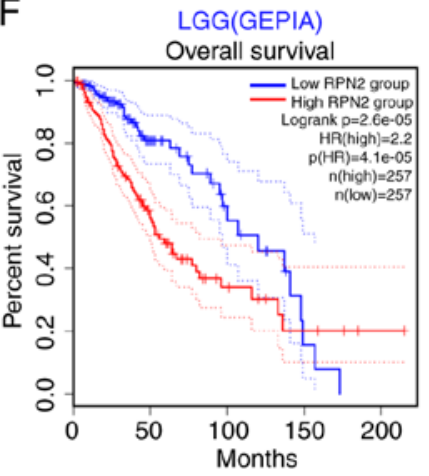

I

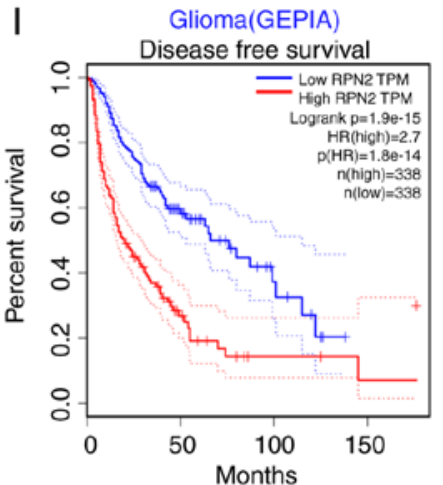

Figure 7. RPN2 overexpression is associated with glioma grade and predicts a poor prognosis. (A) RPN2 expression level in gliomas with distinct WHO grades from the CGGA database. Kaplan-Meier survival curves for (B) primary and (C) recurrent gliomas with low and high RPN2 expression from the CGGA database. (D) UALCAN analysis of RPN2 expression in LGG patients, including those with grade 2 and 3 tumors. (E) UALCAN analysis of the prognostic significance of RPN2 expression in LGG patients. GEPIA analysis of the association between RPN2 expression and (F) overall survival or (G) disease-free survival for patients with LGG. GEPIA analysis of the association between RPN2 expression and (H) overall survival or (I) disease-free survival for patients with glioma. High levels of RPN2 predicted a significantly worse outcome. ${ }^{* *} \mathrm{P}<0.01,{ }^{* * * *} \mathrm{P}<0.0001$. RPN2, ribophorin II; CGGA, Chinese Glioma Genome Atlas; GEPIA, Gene Expression Profiling Interactive Analysis; HR, hazard ratio; WHO, World Health Organization; LGG, low-grade glioma.

HSP90, which are essential for mtp53 stabilization, enhanced initiation, metastasis and cancer stem cell property acquisition in breast cancer. Our previous experimental data also verified that the knockdown of RPN2 inhibited the Wnt/ $\beta$-catenin pathway in glioma (data not shown); hence, it was hypothesized that miR-422a suppresses glioma growth through the $\mathrm{Wnt} / \beta$-catenin signaling pathway via RPN2. Indeed, the present results revealed that miR-422a markedly disrupted the $\mathrm{Wnt} / \beta$-catenin pathway, whereas restoration of RPN2 in LN229 and U87 cells with miR-422a overexpression could in part abrogate the inhibitory effect of miR-422a. Therefore, to the best of our knowledge, the present study demonstrated for the first time the relationship between miR-422a, RPN2 and the Wnt pathway in glioma.

The miRNA/mRNA/signaling pathway is a complicated regulatory network that is a critical molecular mechanism for GBM recurrence, TMZ resistance and EMT (41-43). miR-422a negatively modulates the EGFR/MEK/ERK signaling pathway by targeting the mediator complex subunit (Med19) (44). Additionally, miR-422a serves as a tumor suppressor through the SULF2-mediated TGF- $\beta$ /SMAD signaling pathway in non-small cell lung cancer (45). Furthermore, miR-422a inhibits the PI3K/AKT pathway by directly targeting PIK3CA and AKT1 in glioma and colorectal cancer, respectively $(14,46)$. Combined with the present data on Wnt signaling pathway regulation by miR-422a, the data suggested that miR-422a may be a crucial anticancer gene that acts through multiple signaling pathways. In addition, Huang et al (47) reported that RPN2 promotes metastasis and suppresses autophagy via STAT3 and NF- $\mathrm{B}$ signaling pathways in hepatocellular carcinoma. These reports reflect the complexity of the signal transduction pathways mediated by 
miR-422a that are implicated in cancer progression, including glioma. However, the specific regulatory mechanism between miR-422a and other pathways, such as NF/KB and STAT3, is worthy of further study.

Mounting evidence has demonstrated that competing endogenous RNA (ceRNA) networks play significant roles in tumor biology. A variety of miRNAs are regulated by other non-coding RNAs, such as circular RNAs (circRNAs) and long non-coding RNAs (lncRNAs) $(6,48)$. For instance, Hong et al (12) reported that circCRIM1 competitively sponges miR-422a to block the inhibitory effect of miR-422a on FOXQ1 and subsequently contributes to nasopharyngeal carcinoma cell metastasis, EMT and docetaxel chemoresistance. Zhou et al (49), demonstrated that lncRNAD63785 acts as a ceRNA of miR-422a and enhances chemoresistance by retarding miR-422a-dependent suppression of MEF2D. However, more circRNAs or lncRNAs sponging miR-422a have been identified and validated $(50,51)$, which may be of significance in understanding the in-depth molecular mechanism of glioma initiation and progression, and combined treatment responses.

In conclusion, the present study confirmed that ectopic miR-422a expression suppresses GBM tumorigenesis and promotes apoptosis by regulating the $\mathrm{Wnt} / \beta$-catenin signaling pathway, and that RPN2 plays significant roles in the miR-422a-mediated effect on tumor growth and Wnt pathway regulation as a direct functional target of miR-422a. These results revealed a novel miR-422a/RPN2/Wnt/ $\beta$-catenin axis in glioma, representing a potential candidate target for GBM therapy.

\section{Acknowledgements}

Not applicable.

\section{Funding}

The study was supported by the Foundation of Tianjin Science and Technology Committee (grant nos. 14JCZDJC35600 and 12ZCDZSY17700), the National Key Technology Support Program (grant nos. 2014BAI04B00 and 2015BAI03B05) and the National Natural Science Fund of China (grant no. 81671169 ).

\section{Availability of data and materials}

The datasets used and/or analysed during the present study are available from the corresponding author on reasonable request.

\section{Authors' contributions}

JS, ZC and JW conceived and designed the experiments. JS, JX, LM and CW performed the in vitro experiments. JS and QW analyzed the data and prepared the figures. WF, JX, $\mathrm{XZ}$ and FT collected specimens and performed the animal experiments. JS and ZC drafted the manuscript. JS, JX, ZC, QW and JW interpreted the data, reviewed and revised the manuscript. All authors have read and approved the final manuscript.

\section{Ethics approval and consent to participate}

The present study was reviewed and approved by Institutional Review Board of Tianjin Huanhu Hospital (Tianjin, China; approval no. CK19-190318), and written informed consent was obtained from all participating patients. All experimental procedures involving the use of animals were approved by the Animal Ethical and Welfare Committee of Tianjin Huanhu Hospital of Nankai University (Tianjin, China; approval no. SYXK2019-001).

\section{Patient consent for publication}

Not applicable.

\section{Competing interests}

The authors declare that they have no competing interests.

\section{References}

1. Aldape K, Zadeh G, Mansouri S, Reifenberger G and von Deimling A: Glioblastoma: Pathology, molecular mechanisms and markers. Acta Neuropathol 129: 829-848, 2015.

2. Kanu OO, Hughes B, Di C, Lin N, Fu J, Bigner DD, Yan H and Adamson C: Glioblastoma multiforme oncogenomics and signaling pathways. Clin Med Oncol 3: 39-52, 2009.

3. Cloughesy TF, Cavenee WK and Mischel PS: Glioblastoma: From molecular pathology to targeted treatment. Annu Rev Pathol 9: 1-25, 2014.

4. Carlsson SK, Brothers SP and Wahlestedt C: Emerging treatment strategies for glioblastoma multiforme. EMBO Mol Med 6: 1359-1370, 2014.

5. Lapointe S, Perry A and Butowski NA: Primary brain tumours in adults. Lancet 392: 432-446, 2018.

6. Chan JJ and Tay Y: Noncoding RNA:RNA regulatory networks in cancer. Int J Mol Sci 19: 1310, 2018.

7. Floyd D and Purow B: Micro-masters of glioblastoma biology and therapy: Increasingly recognized roles for microRNAs. Neuro Oncol 16: 622-627, 2014.

8. Slack FJ and Chinnaiyan AM: The role of Non-coding RNAs in oncology. Cell 179: 1033-1055, 2019.

9. Zhang J, Yang Y, Yang T, Yuan S, Wang R, Pan Z, Yang Y, Huang G, Gu F, Jiang B, et al: Double-negative feedback loop between microRNA-422a and forkhead box (FOX)G1/Q1/E1 regulates hepatocellular carcinoma tumor growth and metastasis. Hepatology 61: 561-573, 2015.

10. Wu L, Hu B, Zhao B, Liu Y, Yang Y, Zhang L and Chen J: Circulating microRNA-422a is associated with lymphatic metastasis in lung cancer. Oncotarget 8: 42173-42188, 2017.

11. Zheng G, Du L, Yang X, Zhang X, Wang L, Yang Y, Li J and Wang C: Serum microRNA panel as biomarkers for early diagnosis of colorectal adenocarcinoma. Br J Cancer 111: 1985-1992, 2014.

12. Hong X, Liu N, Liang Y, He Q, Yang X, Lei Y, Zhang P, Zhao Y, He S, Wang Y, et al: Circular RNA CRIM1 functions as a ceRNA to promote nasopharyngeal carcinoma metastasis and docetaxel chemoresistance through upregulating FOXQ1. Mol Cancer 19: 33, 2020.

13. Du S, Wang S, Zhang F and Lv Y: SKP2, positively regulated by circ_ODC $1 / \mathrm{miR}-422 \mathrm{a}$ axis, promotes the proliferation of retinoblastoma. J Cell Biochem 121: 322-331, 2020.

14. Liang H, Wang R, Jin Y, Li J and Zhang S: MiR-422a acts as a tumor suppressor in glioblastoma by targeting PIK3CA. Am J Cancer Res 6: 1695-1707, 2016.

15. Lemjabbar-Alaoui H, McKinney A, Yang YW, Tran VM and Phillips JJ: Glycosylation alterations in lung and brain cancer. Adv Cancer Res 126: 305-344, 2015.

16. Ono M, Tsuda H, Kobayashi T, Takeshita F, Takahashi RU, Tamura K, Akashi-Tanaka S, Moriya T, Yamasaki T, Kinoshita T, et al: The expression and clinical significance of ribophorin II (RPN2) in human breast cancer. Pathol Int 65: 301-308, 2015. 
17. Veillon L, Fakih C, Abou-El-Hassan H, Kobeissy F and Mechref Y: Glycosylation changes in brain cancer. ACS Chem Neurosci 9: 51-72, 2018.

18. Fujimoto D, Goi T, Koneri K and Hirono Y: RPN2 is effective biomarker to predict the outcome of combined chemotherapy docetaxel and cisplatin for advanced gastric cancer. Oncotarget 9 : 15208-15218, 2018.

19. Fujiwara T, Takahashi RU, Kosaka N, Nezu Y, Kawai A, Ozaki T and Ochiya T: RPN2 gene confers osteosarcoma cell malignant phenotypes and determines clinical prognosis. Mol Ther Nucleic Acids 3: e189, 2014.

20. Zhang J, Yan B, Spath SS, Qun H, Cornelius S, Guan D, Shao J, Hagiwara K, Van Waes C, Chen Z, et al: Integrated transcriptional profiling and genomic analyses reveal RPN2 and HMGB1 as promising biomarkers in colorectal cancer. Cell Biosci 5: 53, 2015.

21. Zhao Z, Meng F, Wang W, Wang Z, Zhang C and Jiang T: Comprehensive RNA-seq transcriptomic profiling in the malignant progression of gliomas. Sci Data 4: 170024, 2017.

22. Tang Z, Kang B, Li C, Chen T and Zhang Z: GEPIA2: An enhanced web server for large-scale expression profiling and interactive analysis. Nucleic Acids Res 47 (W1): W556-W560, 2019.

23. Chandrashekar DS, Bashel B, Balasubramanya SAH Creighton CJ, Ponce-Rodriguez I, Chakravarthi BVSK and Varambally S: UALCAN: A portal for facilitating tumor subgroup gene expression and survival analyses. Neoplasia 19 649-658, 2017.

24. Livak KJ and Schmittgen TD: Analysis of relative gene expression data using real-time quantitative PCR and the 2(-Delta Delta C(T)) method. Methods 25: 402-408, 2001.

25. Sun J, Jia Z, Li B, Zhang A, Wang G, Pu P, Chen Z, Wang Z and Yang W: MiR-19 regulates the proliferation and invasion of glioma by RUNX3 via $\beta$-catenin/Tcf-4 signaling. Oncotarget 8: 110785-110796, 2017.

26. He Z, Li Z, Zhang X, Yin K, Wang W, Xu Z, Li B, Zhang L, Xu J, Sun G, et al: MiR-422a regulates cellular metabolism and malignancy by targeting pyruvate dehydrogenase kinase 2 in gastric cancer. Cell Death Dis 9: 505, 2018.

27. Zhang H, He QY, Wang GC, Tong DK, Wang RK, Ding WB Li C, Wei Q, Ding C, Liu PZ, et al: miR-422a inhibits osteosarcoma proliferation by targeting BCL2L2 and KRAS. Biosci Rep 38: BSR20170339, 2018.

28. Li R, Gao K, Luo H, Wang X, Shi Y, Dong Q, Luan W and You Y: Identification of intrinsic subtype-specific prognostic microRNAs in primary glioblastoma. J Exp Clin Cancer Res 33: 9, 2014

29. Maris C, D'Haene N, Trepant AL, Le Mercier M, Sauvage S, Allard J, Rorive S, Demetter P, Decaestecker C and Salmon I: IGF-IR: A new prognostic biomarker for human glioblastoma. $\mathrm{Br}$ J Cancer 113: 729-737, 2015.

30. Wang H, Tang C, Na M, Ma W, Jiang Z, Gu Y, Ma G, Ge H Shen $\mathrm{H}$ and Lin Z: miR-422a inhibits glioma proliferation and invasion by targeting IGF1 and IGF1R. Oncol Res 25: 187-194, 2017.

31. De Souza R, Zahedi P, Badame RM, Allen C and PiquetteMiller M: Chemotherapy dosing schedule influences drug resistance development in ovarian cancer. Mol Cancer Ther 10: $1289-1299,2011$

32. Honma K, Iwao-Koizumi K, Takeshita F, Yamamoto $Y$ Yoshida T, Nishio K, Nagahara S, Kato K and Ochiya T: RPN2 gene confers docetaxel resistance in breast cancer. Nat Med 14 939-948, 2008

33. Kurashige J, Watanabe M, Iwatsuki M, Kinoshita K, Saito S, Nagai Y, Ishimoto T, Baba Y, Mimori K and Baba H: RPN2 expression predicts response to docetaxel in oesophageal squamous cell carcinoma. Br J Cancer 107: 1233-1238, 2012.

34. Zhang H, Jiang H, Zhang H, Liu J, Hu X and Chen L: Ribophorin II potentiates P-glycoprotein- and ABCG2-mediated multidrug resistance via activating ERK pathway in gastric cancer. Int J Biol Macromol 128: 574-582, 2019.
35. Koshkin PA, Chistiakov DA, Nikitin AG, Konovalov AN, Potapov AA, Usachev DY, Pitskhelauri DI, Kobyakov GL, Shishkina LV and Chekhonin VP: Analysis of expression of microRNAs and genes involved in the control of key signaling mechanisms that support or inhibit development of brain tumors of different grades. Clin Chim Acta 430: 55-62, 2014.

36. Stavrovskaya AA, Shushanov SS and Rybalkina EY: Problems of glioblastoma multiforme drug resistance. Biochemistry (Mosc) 81: 91-100, 2016

37. Zhang K, Zhang J, Han L, Pu P and Kang C: Wnt/beta-catenin signaling in glioma. J Neuroimmune Pharmacol 7: 740-749, 2012.

38. He L, Zhou H, Zeng Z, Yao H, Jiang W and Qu H: Wnt/ $\beta$-catenin signaling cascade: A promising target for glioma therapy. J Cell Physiol 234: 2217-2228, 2019.

39. Lim JC, Kania KD, Wijesuriya H, Chawla S, Sethi JK, Pulaski L, Romero IA, Couraud PO, Weksler BB, Hladky SB and Barrand MA: Activation of beta-catenin signalling by GSK-3 inhibition increases p-glycoprotein expression in brain endothelial cells. J Neurochem 106: 1855-1865, 2008.

40. Takahashi RU, Takeshita F, Honma K, Ono M, Kato K and Ochiya T: Ribophorin II regulates breast tumor initiation and metastasis through the functional suppression of GSK3 $\beta$. Sci Rep 3: 2474, 2013.

41. Low SY, Ho YK, Too HP, Yap CT and Ng WH: MicroRNA as potential modulators in chemoresistant high-grade gliomas. J Clin Neurosci 21: 395-400, 2014.

42. Novakova J, Slaby O, Vyzula R and Michalek J: MicroRNA involvement in glioblastoma pathogenesis. Biochem Biophys Res Commun 386: 1-5, 2009.

43. Xin S, Huang K and Zhu XG: Non-coding RNAs: Regulators of glioma cell epithelial-mesenchymal transformation. Pathol Res Pract 215: 152539, 2019.

44. Zhang X, Gao D, Fang K, Guo Z and Li L: Med19 is targeted by miR-101-3p/miR-422a and promotes breast cancer progression by regulating the EGFR/MEK/ERK signaling pathway. Cancer Lett 444: 105-115, 2019.

45. Li WQ, Zhang JP, Wang YY, Li XZ and Sun L: MicroRNA-422a functions as a tumor suppressor in non-small cell lung cancer through SULF2-mediated TGF- $\beta$ /SMAD signaling pathway. Cell Cycle 18: 1727-1744, 2019

46. Wei WT, Nian XX, Wang SY, Jiao HL, Wang YX, Xiao ZY, Yang RW, Ding YQ, Ye YP and Liao WT: miR-422a inhibits cell proliferation in colorectal cancer by targeting AKT1 and MAPK1. Cancer Cell Int 17: 91, 2017.

47. Huang L, Jian Z, Gao Y, Zhou P, Zhang G, Jiang B and Lv Y: RPN2 promotes metastasis of hepatocellular carcinoma cell and inhibits autophagy via STAT3 and NF-KB pathways. Aging (Albany NY) 11: 6674-6690, 2019.

48. Dragomir M, Mafra ACP, Dias SMG, Vasilescu C and Calin GA: Using microRNA networks to understand cancer. Int J Mol Sci 19: 1871, 2018

49. Zhou Z, Lin Z, He Y, Pang X, Wang Y, Ponnusamy M, Ao X, Shan P, Tariq MA, Li P and Wang J: The long noncoding RNA D63785 regulates chemotherapy sensitivity in human gastric cancer by targeting miR-422a. Mol Ther Nucleic Acids 12: 405-419, 2018

50. Wang R, Zhang S, Chen X, Li N, Li J, Jia R, Pan Y and Liang H: CircNT5E Acts as a Sponge of miR-422a to promote glioblastoma tumorigenesis. Cancer Res 78: 4812-4825, 2018.

51. Wei F, Yang L, Jiang D, Pan M, Tang G, Huang M and Zhang J Long noncoding RNA DUXAP8 contributes to the progression of hepatocellular carcinoma via regulating miR-422a/PDK2 axis. Cancer Med 9: 2480-2490, 2020.

This work is licensed under a Creative Commons Attribution-NonCommercial-NoDerivatives 4.0 International (CC BY-NC-ND 4.0) License. 